\title{
Applicability of Aegilops tauschii drought tolerance traits to breeding of hexaploid wheat
}

\author{
Quahir Sohail'), Tomoe Inoue $^{\text {2), Hiroyuki Tanaka }}{ }^{3)}$, Amin Elsadig Eltayeb'), Yoshihiro Matsuoka ${ }^{4)}$ \\ and Hisashi Tsujimoto*1) \\ 1) Laboratory of Molecular Breeding, Arid Land Research Center, Tottori University, 1390 Hamasaka, Tottori 680-0001, Japan \\ 2) Laboratory of Plant Ecophysiology, Arid Land Research Center, Tottori University, 1390 Hamasaka, Tottori 680-0001, Japan \\ 3) Laboratory of Plant Genetics, Faculty of Agriculture, Tottori University, 4-101 Minami, Tottori 680-8553, Japan \\ 4) Department of Bioscience, Fukui Prefectural University, Matsuoka, Eiheiji, Yoshida, Fukui 910-1195, Japan
}

\begin{abstract}
Few genes are available to develop drought-tolerant bread wheat (Triticum aestivum L.) cultivars. One way to enhance bread wheat's genetic diversity would be to take advantage of the diversity of wild species by creating synthetic hexaploid wheat (SW) with the genomic constitution of bread wheat. In this study, we compared the expression of traits encoded at different ploidy levels and evaluated the applicability of Aegilops tauschii drought-related traits using 33 Ae. tauschii accessions along with their corresponding SW lines under well-watered and drought conditions. We found wide variation in Ae. tauschii, and even wider variation in the SW lines. Some SW lines were more drought-tolerant than the standard cultivar Cham 6. Aegilops tauschii from some regions gave better performing SW lines. The traits of Ae. tauschii were not significantly correlated with their corresponding SW lines, indicating that the traits expressed in wild diploid relatives of wheat may not predict the traits that will be expressed in SW lines derived from them. We suggest that, regardless of the adaptability and performance of the Ae. tauschii under drought, production of SW could probably result in genotypes with enhanced trait expression due to gene interactions, and that the traits of the synthetic should be evaluated in hexaploid level.
\end{abstract}

Key Words: wheat, Aegilops tauschii, synthetic hexaploid wheat, wild relatives of wheat.

\section{Introduction}

Feeding the world's growing population is becoming a huge challenge. Food prices, food shortages, and malnutrition are increasing (Webb 2010). Climate change is expected to worsen the current situation through its effects on global agricultural systems (Battisti and Naylor 2009, Bloem et al. 2010). Severe scenarios such as significant drying in some regions, leading to an increased frequency and severity of extreme droughts, are expected (Allen et al. 2010, Carnicer et al. 2010, Soussana et al. 2010). Bread wheat (Triticum aestivum L.) is affected by periodic drought in around $50 \%$ of its cultivated area (Rajaram 2001), and its productivity is often limited because there is insufficient water to maximize biomass and complete grain-filling (Aprile et al. 2009).

Few genes have been identified in bread wheat that can be used to develop drought-tolerant cultivars. Millions of years ago, a natural hybridization may have occurred between the wild diploid wheat Triticum urartu (genome AA) and the wild grass Aegilops speltoides (SS), resulting in the

Communicated by M. Iwanaga

Received July 4, 2011. Accepted August 5, 2011.

*Corresponding author (e-mail: tsujim@alrc.tottori-u.ac.jp)
AABB tetraploid emmer wheat, Triticum dicoccoides (Mujeeb-Kazi et al. 1996). Later, the domestication of emmer wheat led to the evolution of durum wheat, Triticum durum $(2 n=28, \mathrm{AABB})$, which was in turn hybridized with the diploid wild goat grass Aegilops tauschii (genome DD) to produce hexaploid bread wheat, Triticum aestivum (AABBDD) about 8000 to 10000 years ago (Feldman 2001, Helbaek 1959, Kihara 1944, Mujeeb-Kazi et al. 1996). Although considerable genetic diversity was preserved in the land races grown by farmers since ancient times, before the spread of modern cultivars with a narrow genetic base, modern wheat breeders have increasingly narrowed this genetic diversity by emphasizing pure-line cultivars. As increasing emphasis was placed on pure lines with higher yields during the Green Revolution, considerable genetic diversity was lost.

Improving wheat drought tolerance through selection and breeding requires a reasonably high level of heritable variation among wheat genotypes or the incorporation of variation from wild relatives, which may serve as a rich source of appropriate genetic variation (Ashraf 2010). As a widely adaptable direct ancestor of bread wheat, Ae. tauschii appears to be the most desirable species for wheat improvement among the more than 300 wild species in the tribe 
Triticeae. This species grows on sandy seashores, at the margins of deserts, on stony hills, in grasslands and wastelands, along roadsides, and even in humid temperate forests (van Slageren 1994). Many useful sources of disease and pest resistance are available in this species (Zaharieva et al. 2001). The genetic diversity within the DD genome of Ae. tauschii is much higher than that of bread wheat (Naghavi and Mardi 2010, Reif et al. 2005). The DD genome is also a rich source of resistance to various biotic and abiotic stresses and could therefore contribute to the improvement of wheat cultivars (Assefa and Fehrmann 2000, Colmer et al. 2006, Cox et al. 1994). Many allohexaploid wheat lines can be produced by artificial hybridization between tetraploid wheat and Ae. tauschii, and the resulting amphidiploid lines are called "resynthesized" or "synthetic hexaploid" wheat (SW) (Mujeeb-Kazi et al. 1996).

However, most breeders use only a limited number of Ae. tauschii lines due to the difficulties associated with creating many interspecific hybrids in conventional breeding programs and due to its weedy morphology, which discourages breeders. Even the SW which has same ploidy as the common wheat, the morphology is quite different from that of hexaploid wheat, and it is difficult to deduce the performance of some traits from SW in hexaploid wheat. Although Ae. tauschii and SW lines are agronomically inferior to modern wheat cultivars, they may possess superior genes that provide important resources for expanding the available genetic variation in bread wheat. SW lines are therefore being used to transfer exotic genes into bread wheat, and crosses between elite common wheat cultivars and SW lines have resulted in lines with improved drought adaptation. It is estimated that one-third of all new advanced bread wheat lines produced by the International Center for the Improvement of Wheat and Maize (CIMMYT) for use in irrigated and low-rainfall areas are SW derivatives (van Ginkel and Ogbonnaya 2007).

Biomass, biomass-related traits, and vegetative growth rates can be used as indicators of drought tolerance (Entz and Fowler 1990), and they are significantly correlated with grain yield under drought conditions (van Ginkel et al. 1998). Various physiological parameters such as the photosynthetic rate, transpiration rate, leaf water potential and root and shoot dry weights are not only important criteria to select for drought tolerance, but also reflect photosynthate production during certain growth stages (Shirazi et al. 2010).

We performed the present study (1) to investigate whether the morphological and physiological traits of Ae. tauschii related to drought adaptation could be expressed at the hexaploid level, (2) to study the usefulness of Ae. tauschii as a tool for predicting the performance of SW lines and (3) to examine whether the drought-related traits of the SW lines were correlated with the respective traits of their wild parents. To accomplish these goals, we analyzed the expression of several drought-related traits encoded in the DD genome of 33 Ae. tauschii accessions and their corresponding SW lines.

\section{Materials and Methods}

\section{Plant materials}

We used 33 Ae. tauschii accessions and 33 SW lines obtained from crossing these lines with $T$. durum cv. Langdon (Matsuoka and Nasuda 2004, Matsuoka et al. 2007). The SW lines were found to be stable (Takumi et al. 2009). Two varieties (T. durum cv. Langdon and T. aestivum cv. Cham 6 ) were included as checks. Cham 6 is drought tolerant bread wheat cultivar with synthetic wheat in its pedigree developed in ICARDA (Table 1). The Ae. tauschii accessions were collected from five groups of locations: West Asia (Armenia, Syria and Turkey), Iran, Georgia, Central Asia (Afghanistan, Kazakhstan, Kyrgyzstan, India’s Jammu and

Table 1. Aegilops tauschii accessions and the corresponding synthetic hexaploid wheat (SW) lines used in this study

\begin{tabular}{|c|c|c|c|c|}
\hline $\begin{array}{l}\text { Serial } \\
\text { no. }\end{array}$ & Accession & Country & $\begin{array}{l}\text { Ae. tauschii } \\
\text { reference. no. }\end{array}$ & $\begin{array}{c}\text { Synthetic } \\
\text { wheat } \\
\text { reference no. }\end{array}$ \\
\hline 1 & AE 1090 & Kazakhstan & $\mathrm{T} 1$ & SW1 \\
\hline 2 & AE 454 & Georgia & $\mathrm{T} 2$ & SW2 \\
\hline 3 & AE 929 & Georgia & T3 & SW3 \\
\hline 4 & AT 55 & China & T4 & SW4 \\
\hline 5 & AT 76 & China & T5 & SW5 \\
\hline 6 & AT 80 & China & T6 & SW6 \\
\hline 7 & IG 126387 & $\begin{array}{l}\text { Turkmenistan } \\
\text { (Ashkhabad) }\end{array}$ & $\mathrm{T} 7$ & SW7 \\
\hline 8 & IG 131606 & Kyrgyzstan (Talas) & T9 & SW9 \\
\hline 9 & IG 47259 & Syria (Raqqa) & T11 & SW11 \\
\hline 10 & IG 48042 & $\begin{array}{l}\text { India } \\
\qquad \text { (Jammu \& Kashmir) }\end{array}$ & T12 & SW12 \\
\hline 11 & KU-2039 & Afghanistan & T14 & SW14 \\
\hline 12 & KU-2069 & Iran & T15 & SW15 \\
\hline 13 & KU-2074 & Iran & T16 & SW16 \\
\hline 14 & KU-2076 & Iran & T18 & SW18 \\
\hline 15 & KU-2078 & Iran & T19 & SW19 \\
\hline 16 & KU-2079 & Iran & $\mathrm{T} 20$ & SW20 \\
\hline 17 & KU-20-8 & Iran & $\mathrm{T} 21$ & SW21 \\
\hline 18 & KU-2080 & Iran & $\mathrm{T} 22$ & SW22 \\
\hline 19 & KU-2088 & Iran & $\mathrm{T} 23$ & SW23 \\
\hline 20 & KU-2103 & Iran & T33 & SW33 \\
\hline 21 & KU-2109 & Iran & $\mathrm{T} 37$ & SW37 \\
\hline 22 & KU-2111 & Iran & T38 & SW38 \\
\hline 23 & KU-2132 & Turkey & T41 & SW41 \\
\hline 24 & KU-2136 & Turkey & T42 & SW42 \\
\hline 25 & KU-2155 & Iran & T44 & SW44 \\
\hline 26 & KU-2158 & Iran & T46 & SW46 \\
\hline 27 & KU-2160 & Iran & T48 & SW48 \\
\hline 28 & KU-2816 & Armenia & T49 & SW49 \\
\hline 29 & KU-2826 & Georgia & T50 & SW50 \\
\hline 30 & KU-2829A & Georgia & T52 & SW52 \\
\hline 31 & PI 476874 & Afghanistan & T53 & SW53 \\
\hline 32 & PI 499262 & China (Xinjian) & T54 & SW54 \\
\hline 33 & PI 508262 & China (Xinjian) & T55 & SW55 \\
\hline 34 & Cham 6 & ICARDA cultivar & & \\
\hline 35 & Langdon & $\begin{array}{l}\text { Triticum durum } \\
\quad \text { (parent of all SW) }\end{array}$ & & \\
\hline
\end{tabular}


Kashmir state, and Turkmenistan), and China. Cham 6 is a drought-tolerant bread wheat cultivar developed by the International Center for Agricultural Research in the Dry Areas (ICARDA).

\section{Experimental conditions}

All accessions were grown in pots under fully controlled conditions, with a day length of $14 \mathrm{~h}$, a light intensity of 80000 lux, a temperature regime of $25^{\circ} \mathrm{C}$ day $/ 20^{\circ} \mathrm{C}$ night and a relative humidity of $50 \%$ day $/ 60 \%$ night. Trials were performed at the Arid Land Research Center, Tottori University, Japan. To break seed dormancy, we first kept the dry seeds at $4^{\circ} \mathrm{C}$ for $48 \mathrm{~h}$. Seeds that did not germinate were treated with $1 \% \mathrm{H}_{2} \mathrm{O}_{2}$ and kept at $4^{\circ} \mathrm{C}$ for a further $16 \mathrm{~h}$; then, fresh $\mathrm{H}_{2} \mathrm{O}_{2}$ was added and the seeds were kept at $25^{\circ} \mathrm{C}$ for a further $30 \mathrm{~h}$. Five seedlings from each accession were transferred into plastic pots containing $1 \mathrm{~kg}$ of dune sand from Tottori City, Japan, and were later thinned to two per pot. Three inorganic fertilizer compounds were added to each pot: $\mathrm{N}: \mathrm{P}: \mathrm{K}(13: 6.5: 12.5), \mathrm{Ca}: \mathrm{Mg}(21: 0.6)$ and $\mathrm{Mg}: \mathrm{Mn}: \mathrm{B}(8.4: 0.3: 0.3)$ at 858,1431 and $572 \mathrm{mg} /$ pot, respectively. The 66 accessions were grown under either well-watered or drought conditions in a factorial experiment using a completely randomized design with three replicates. The pot weight was recorded daily, and an amount of water equal to the loss was added during the initial establishment period. The volumetric moisture content of the soil was initially maintained at 9 to $12 \%$. Seventeen days after sowing, drought was induced in half of the plants by allowing the moisture content to fall to between 6 and $8 \%$, while the wellwatered treatment was maintained as described above.

\section{Morphological and physiological measurements}

Physiological parameters were recorded using the uppermost fully expanded leaf of the 30- and 37-day-old plants. Photosynthesis (the net photosynthetic rate), stomatal conductance, intercellular $\mathrm{CO}_{2}$ concentration, and transpiration rate were measured using an LI-6400 portable photosynthesis system (LI-COR, Lincoln, NE, USA). Leaf water potential (LWP) in 42-day-old plants was measured 19 to $20 \mathrm{~h}$ after irrigation using a model 600 pressure chamber (PMS Instrument Company, Albany, OR, USA). Chlorophyll content was estimated using a Minolta SPAD-502 leaf chlorophyll meter (Minolta Camera Co. Ltd., Osaka, Japan). Plants were harvested on day 52 and the roots and shoots were collected separately, dried at $65^{\circ} \mathrm{C}$ for 2 days, weighed, and then used to calculate the total dry weight (TDW), root dry weight (RDW), shoot dry weight (SDW) and the rootshoot ratio (RSR). Water-use efficiency (WUE) was calculated as the weight $(\mathrm{g})$ of dry matter produced per $\mathrm{kg}$ of water applied. The partitioning of dry matter to the roots (PDR, \%) was calculated as the root weight divided by the total weight, then multiplied by $100 \%$.

\section{Statistical analysis}

Data were analyzed using ANOVA for a completely randomized design. Fisher's protected LSD was used to test differences among genotypes and differences within genotypes between the well-watered and drought conditions. Genotypic variance of the SW lines (from the ANOVA results) was standardized (SW values were divided by Ae. tauschii and multiplied by 100) to produce a value of 100 for Ae. tauschii, thereby facilitating comparisons with the SW lines.

Correlations of the morphological and physiological traits were estimated based on Pearson correlation coefficient.

Euclidean measure of diversity was used to compare the diversity of the Ae. tauschii and SW lines. The Euclidean distance is the square root of the sum of squared differences between the different trait values for a pair of genotypes. As the data had different units, the data were statistically standardized (by subtracting the mean form the data and then dividing it by the standard deviation) and the distances were calculated using the following formula (Teknomo 2011):

$$
D_{i j}=\sqrt{\sum_{k=1}^{n}\left(\left(X_{i k}-X_{j k}\right)^{2}\right)}
$$

Where Dij represents the Euclidean distance between $i$ and $j$ genotypes, $k$ represents the traits form 1 to $\mathrm{n}$ and $X_{i k}$ represents the kth trait of $i t h$ genotype and $X_{j k}$ represent the $k t h$ trait of the $j t h$ genotype. From the standardized distances, which fall within the range $[0,1]$, we calculated the mean, maximum, minimum, variance and standard deviation $(\mathrm{SD})$ of the $528[=(33 \times[33-1]) / 2]$ distances for all Ae. tauschii and SW lines under well-watered and drought conditions.

To test the significance of any differences between the mean distances (the 528 paired values) for the Ae. tauschii and SW lines under well-watered and drought conditions separately, we used a $t$-test based on unequal variances because the variances of the two groups under each water regime were significantly different based on the results of an $F$-test. The inequality of the variances of the distances also showed significant diversity between the two groups of genotypes.

$$
\begin{aligned}
& F=\frac{\text { Variance } S W}{\text { Variance } A T} \\
& t=\frac{\text { Mean } S W=\text { Mean } A T}{\sqrt{(\text { Pooled variance } / 528)}}
\end{aligned}
$$

Where SW represents the value for the SW lines and AT represents the value for the Ae.tauschii lines.

\section{Results}

The SW lines inherited useful morphological and physiological variation

We observed a high degree of variation for most morphological and physiological traits studied in the Ae. tauschii and SW lines under both well-watered and drought conditions. 
Table 2. Mean squares (MS) from the ANOVA results for morphological and physiological traits of the Ae. tauschii lines and their corresponding SW lines grown under well-watered and drought conditions

\begin{tabular}{|c|c|c|c|c|c|c|c|c|c|c|c|c|c|c|}
\hline Trait $^{b}$ & $\begin{array}{c}\text { Water } \\
\text { regime }^{a}\end{array}$ & Group & $\begin{array}{c}\text { Photosyn- } \\
\text { thesis }\end{array}$ & $\begin{array}{l}\text { Stomatal } \\
\text { conduc- } \\
\text { tance }\end{array}$ & $\begin{array}{c}\text { Intercellu- } \\
\text { lar } \mathrm{CO}_{2}\end{array}$ & $\begin{array}{l}\text { Transpira- } \\
\text { tion rate }\end{array}$ & $\begin{array}{l}\text { SPAD } \\
\text { reading }\end{array}$ & LWP & RDW & SDW & TDW & RSR & PDR & WUE \\
\hline \multirow{4}{*}{$\begin{array}{l}\text { Geno- } \\
\text { types (G) }\end{array}$} & WWC & $\mathrm{AT}^{d}$ & $38.779 * *$ & $0.028 \mathrm{~ns}$ & $93632 * *$ & $8.426^{*}$ & $72.94 * *$ & $10.05 * *$ & $1.791 * *$ & $0.606^{* *}$ & $3.668 * *$ & $0.528 \mathrm{~ns}$ & $167.38 \mathrm{~ns}$ & $1.082 * *$ \\
\hline & & SW & $27.940 * *$ & $0.031 * *$ & $54676 * *$ & $2.600 \mathrm{~ns}$ & $173.16^{* *}$ & $10.35 * *$ & $2.303 * *$ & $0.811^{* *}$ & $5.195 * *$ & $0.349 \mathrm{~ns}$ & $168.39 \mathrm{~ns}$ & $1.823 * *$ \\
\hline & $\mathrm{DC}$ & $\mathrm{AT}$ & $34.166^{* *}$ & $0.037 \mathrm{~ns}$ & $13488^{*}$ & $4.051 \mathrm{~ns}$ & $46.81 * *$ & $16.36^{* *}$ & $0.431 * *$ & $0.114 \mathrm{~ns}$ & $0.702 \mathrm{~ns}$ & $0.573 \mathrm{~ns}$ & $269.63 \mathrm{~ns}$ & $0.461 \mathrm{~ns}$ \\
\hline & & SW & $28.252 \mathrm{~ns}$ & $0.040 \mathrm{~ns}$ & $10339 * *$ & $1.648 \mathrm{~ns}$ & $61.49 * *$ & $18.55^{* *}$ & $1.669^{* *}$ & $0.352 * *$ & $3.019 * *$ & $1.075^{* *}$ & $334.68 * *$ & $1.735^{* *}$ \\
\hline \multirow{4}{*}{ Error } & WWC & $\mathrm{AT}$ & 18.046 & 0.019 & 5976 & 4.534 & 16.16 & 1.97 & 0.635 & 0.186 & 0.797 & 0.614 & 159.34 & 0.239 \\
\hline & & SW & 13.721 & 0.014 & 5243 & 2.279 & 11.92 & 1.49 & 0.413 & 0.177 & 0.814 & 0.353 & 137.17 & 0.281 \\
\hline & DC & AT & 10.064 & 0.035 & 7739 & 2.761 & 17.25 & 7.34 & 0.178 & 0.157 & 0.476 & 0.677 & 221.66 & 0.384 \\
\hline & & SW & 19.317 & 0.035 & 4947 & 1.826 & 21.28 & 3.44 & 0.249 & 0.104 & 0.453 & 0.517 & 136.07 & 0.351 \\
\hline \multirow{4}{*}{$\mathrm{LSD}^{c}$} & WWC & AT & 6.929 & 0.224 & 126 & 3.47 & 6.6 & 2.3 & 1.3 & 0.703 & 1.457 & - & - & 0.798 \\
\hline & & SW & 6.039 & 0.191 & 118 & - & 5.6 & 2.0 & 1.048 & 0.687 & 1.473 & - & - & 0.865 \\
\hline & $\mathrm{DC}$ & $\mathrm{AT}$ & 5.181 & - & 144 & - & 6.8 & 4.4 & 0.689 & - & 1.128 & - & - & - \\
\hline & & SW & 7.171 & - & 115 & - & 7.5 & 3.0 & 0.813 & 0.527 & 1.099 & 1.174 & 19.1 & 0.969 \\
\hline
\end{tabular}

${ }^{a}$ WWC, well-watered conditions; DC, drought conditions.

${ }^{b}$ LWP, leaf water potential; RDW, root dry weight; SDW, shoot dry weight; TDW, total dry weight; RSR, root-shoot ratio; PDR, partitioning of dry matter to roots (\%); WUE, water-use efficiency.

${ }^{c}$ LSD, least significant difference.

${ }^{d}$ AT, Ae. tauschii; SW, synthetic hexaploid wheat;**, $P<0.01 ; *, P<0.05 ;$ ns, not significant.

Under well-watered conditions, highly significant differences were observed in all the studied morphological and physiological traits except for stomatal conductance, transpiration rate, root-shoot ratio, and PDR in the Ae. tauschii and SW lines (Table 2). Neither group differed significantly with regard to the root-shoot ratio and PDR. Although the SW lines showed high and significant differences for stomatal conductance, no differences were observed among the Ae. tauschii accessions. In contrast, only the Ae. tauschii lines showed significant differences in transpiration rate under well-watered conditions. The Ae. tauschii lines transpired more but produced more dry matter per unit water than the SW lines under well-watered conditions. These observations were consistent with the averages of the stomatal conductance, transpiration rate, and TDW values shown in Table 3.

When exposed to drought, large differences were observed between the two groups in the traits related to water use and biomass production. Only the SW group exhibited a highly significant difference for SDW, TDW, RSR, PDR and WUE (Table 2). Moreover, the SW lines had higher averages than the Ae. tauschii lines for these traits (Table 3), which indicates their ability to use water more efficiently for biomass production under drought conditions.

\section{SW lines showed greater drought tolerance than Ae. tauschii lines}

The Ae. tauschii lines had higher RDW, SDW, and TDW under well-watered conditions, but they sustained a greater reduction in these traits under drought conditions (Table 3). The SW group showed better tolerance of drought conditions both in terms of the actual RDW, SDW, and TDW values and in terms of the percent reduction in these values due to drought (Table 3). This was clearly reflected in the RSR and the PDR, which both decreased in Ae. tauschii under drought conditions but increased in the SW group (Table 3). The SW group showed a smaller percent reduction in average TDW (33.8\%) than the Ae. tauschii group (51.8\%) and Langdon (42.6\%), but Cham 6 showed the least reduction (12.4\%). Interestingly, SW5 had $18.4 \%$ greater TDW under drought conditions. Although SW2 had a $29.2 \%$ reduction in TDW under drought conditions, it produced the highest TDW $(3.8 \mathrm{~g} / \mathrm{pot})$ among all the genotypes. Under drought conditions, RDW decreased by $53.0 \%$ in the Ae. tauschii group, versus $30.1 \%$ in the SW group. Despite the general reduction in RDW with drought, Cham 6, SW19 and SW5 produced 105.0, 22.6 and $44.3 \%$ more RDW, respectively.

Although the Ae. tauschii group had higher average photosynthetic rates than the SW group under both well-watered and drought conditions, the SW accessions had a smaller reduction in photosynthetic rate (20.4\% vs. $21.8 \%)$ (Table 3$)$. The standard check line Cham 6 maintained the highest average photosynthetic rate $\left(26.7 \mu \mathrm{mol} \mathrm{m}^{-2} \mathrm{~s}^{-1}\right)$ and sustained the smallest reduction (5.7\%) under drought conditions.

The Ae. tauschii accessions used water more efficiently than the SW accessions under well-watered conditions (Table 3), but exhibited a greater reduction in their average WUE under drought conditions (30.6 and $2.7 \%$, respectively). Cham 6, SW5 and SW6 used water more efficiently (23, 81 and $28 \%$, respectively) under drought conditions than under well-watered conditions (Table 3 ).

The Ae. tauschii group maintained higher transpiration rates and higher stomatal conductance under both conditions, and responded to drought by exhibiting a higher reduction in their transpiration and stomatal conductance than in the SW group (Table 3). Although the chlorophyll content 
Table 3. Consolidated table showing the mean values of the morphological and physiological characteristics of the Ae. tauschii lines and their corresponding SW lines under well-watered and drought conditions

\begin{tabular}{|c|c|c|c|c|c|c|c|c|}
\hline \multirow{2}{*}{ Traits $^{b}$} & \multirow{2}{*}{ Group } & \multirow{2}{*}{ WWC } & \multirow{2}{*}{$\mathrm{DC}$} & \multirow{2}{*}{$\begin{array}{l}\% \text { reduction with } \\
\text { drought }\end{array}$} & \multicolumn{2}{|c|}{$\mathrm{WWC}^{a}$} & \multicolumn{2}{|c|}{$\mathrm{DC}$} \\
\hline & & & & & Min & Max & Min & Max \\
\hline \multirow[t]{2}{*}{ TDW (g per pot) } & $\mathrm{AT}^{c}$ & 3.59 & 1.73 & 51.8 & 1.23 & 6.04 & 0.93 & 2.88 \\
\hline & SW & 3.11 & 2.06 & 33.8 & 0.92 & 5.35 & 0.32 & 3.78 \\
\hline \multirow[t]{2}{*}{ RDW (g per pot) } & $\mathrm{AT}$ & 2.00 & 0.94 & 53.0 & 0.52 & 4.23 & 0.31 & 2.01 \\
\hline & SW & 1.66 & 1.16 & 30.1 & 0.35 & 3.21 & 0.11 & 2.60 \\
\hline \multirow[t]{2}{*}{ SDW (g per pot) } & $\mathrm{AT}$ & 1.59 & 0.80 & 49.7 & 0.57 & 2.38 & 0.50 & 1.39 \\
\hline & SW & 1.44 & 0.90 & 37.5 & 0.57 & 2.33 & 0.12 & 1.73 \\
\hline \multirow[t]{2}{*}{ RSR } & $\mathrm{AT}$ & 1.35 & 1.29 & 4.4 & 0.57 & 2.32 & 0.17 & 2.40 \\
\hline & SW & 1.16 & 1.32 & -13.8 & 0.60 & 1.80 & 0.51 & 3.10 \\
\hline \multirow[t]{2}{*}{ PDR } & $\mathrm{AT}$ & 53.7 & 51.7 & 3.7 & 36.0 & 69.1 & 13.2 & 69.5 \\
\hline & SW & 50.6 & 52.4 & -3.6 & 34.9 & 64.2 & 32.2 & 71.9 \\
\hline \multirow{2}{*}{$\begin{array}{l}\text { Photosynthesis } \\
\qquad\left(\mu \mathrm{mol} \mathrm{m}^{-2} \mathrm{~s}^{-1}\right)\end{array}$} & $\mathrm{AT}$ & 30.7 & 24.0 & 21.8 & 24.8 & 38.6 & 17.4 & 32.7 \\
\hline & SW & 27.0 & 21.5 & 20.4 & 19.6 & 32.9 & 14.8 & 27.9 \\
\hline \multirow[t]{2}{*}{ WUE $\left(\mathrm{g} \mathrm{kg}^{-1}\right)$} & $\mathrm{AT}$ & 2.09 & 1.45 & 30.6 & 0.72 & 3.45 & 0.81 & 2.33 \\
\hline & SW & 1.85 & 1.80 & 2.7 & 0.59 & 3.32 & 0.32 & 3.33 \\
\hline \multirow{2}{*}{$\begin{array}{l}\text { Transpiration rate } \\
\qquad \mathrm{mmol} \mathrm{H}_{2} \mathrm{O} \mathrm{m}^{-2} \mathrm{~s}^{-1}\end{array}$} & $\mathrm{AT}$ & 5.03 & 3.9 & 22.5 & 1.43 & 9.30 & 1.78 & 7.6 \\
\hline & SW & 3.69 & 3.1 & 16.0 & 1.71 & 5.8 & 2.23 & 4.30 \\
\hline \multirow{2}{*}{$\begin{array}{l}\text { Stomatal conduct. } \\
\left(\mathrm{mol} \mathrm{m}^{-2} \mathrm{~s}^{-1}\right)\end{array}$} & $\mathrm{AT}$ & 0.30 & 0.27 & 10.0 & 0.15 & 0.57 & 0.13 & 0.58 \\
\hline & SW & 0.25 & 0.23 & 8.0 & 0.13 & 0.59 & 0.09 & 0.56 \\
\hline \multirow{2}{*}{$\begin{array}{l}\text { Inter cellular } \mathrm{CO}_{2} \\
(\mathrm{ppm})\end{array}$} & $\mathrm{AT}$ & 370 & 253 & 31.6 & 146 & 648 & 153 & 453 \\
\hline & SW & 268 & 211 & 21.3 & 127 & 533 & 109 & 357 \\
\hline \multirow{2}{*}{$\begin{array}{l}\text { Chlorophyll content } \\
\text { (SPAD reading) }\end{array}$} & $\mathrm{AT}$ & 48.1 & 49.3 & -2.5 & 39 & 59.2 & 40.5 & 55.8 \\
\hline & SW & 53.1 & 53.2 & -0.2 & 38.4 & 69.1 & 45.8 & 63.5 \\
\hline \multirow[t]{2}{*}{$\overline{L W P}$} & AT & -8.52 & -10.27 & -20.5 & 5.17 & 14.5 & 6.67 & 16.3 \\
\hline & SW & -7.6 & -9.78 & -28.7 & 4.67 & 10.5 & 4.33 & 16.5 \\
\hline
\end{tabular}

${ }^{a}$ WWC, well-watered conditions; DC, drought conditions.

${ }^{b}$ TDW, total dry weight; RDW, root dry weight; SDW, shoot dry weight; RSR, root-shoot ratio; PDR, partitioning of dry matter to roots (\%); WUE, water-use efficiency; LWP, leaf water potential.

${ }^{c} \mathrm{AT}$, Ae. tauschii; SW, synthetic hexaploid wheat.

in both groups was not greatly affected by drought, the SW group maintained higher chlorophyll contents under both conditions (Table 3). The intercellular $\mathrm{CO}_{2}$ concentration was higher and water potential was lower (more negative) in the Ae. tauschii group than in the SW group under both conditions.

The SW lines had higher genotypic variance than the Ae. tauschii lines

We analyzed the genotypic variance of the two groups of lines based on their morphological and physiological performance, using the ANOVA mean squares of Ae. tauschii for each characteristic as the standard of comparison (with a standardized value of 100 for each characteristic). Although the SW lines showed remarkably less genotypic variance in photosynthetic rate, intercellular $\mathrm{CO}_{2}$ concentration, and transpiration rate under both well-watered and drought conditions (Table 4), the SW lines showed higher genotypic variance in all other parameters except the RSR (i.e., higher variance in stomatal conductance, chlorophyll content, wa- ter potential, RDW, SDW, TDW, PDR and WUE). Interestingly, although the SW lines showed less variation than the Ae. tauschii lines in the RSR under well-watered conditions, they showed higher variation in this parameter under drought conditions.

Greater variation in the SW genotypes based on the Euclidian distance

To study the degrees of variation among the Ae. tauschii and SW lines, we adopted the Euclidian distance, standardized to $[0,1]$ according to the method of Teknomo (2011). The mean Euclidean distance revealed more variation in the SW group than in the Ae. tauschii group under both conditions $(0.539$ compared to 0.486 under well-watered conditions and 0.593 compared to 0.475 under drought conditions). Within the Ae. tauschii group, greater variation was observed under well-watered conditions (0.486) than under drought conditions (0.475). In contrast, the SW accessions showed more variation under drought conditions (0.593) than under well-watered conditions (0.539). 
Correlations among the morphological and physiological traits

Under well-watered conditions, the transpiration rate and intercellular $\mathrm{CO}_{2}$ concentration were significantly correlated with the photosynthetic rate in the Ae. tauschii group but not in the SW group (Table 6). Similarly, the three biomass traits (RDW, SDW and TDW) were correlated with the photosynthetic rate in the Ae. tauschii group but not in the SW group. Although the RSR and PDR were not significantly correlated with LWP in the Ae. tauschii group, they were significantly correlated in the SW group (Table 6). In both groups, WUE was significantly correlated with the intercellular $\mathrm{CO}_{2}$ concentration, transpiration rate, chlorophyll content, and the three biomass traits (Table 6). For all other pairs of parameters, the correlations showed similar patterns for the morphological and physiological traits within SW or Ae. tauschii.

Under drought conditions, the photosynthetic rate was significantly correlated with the chlorophyll content, RDW, and the root-shoot ratio in the Ae. tauschii group but not in the SW group (Table 7). Although stomatal conductance was not significantly correlated with any trait in the Ae.tauschii group, it was significantly correlated with the chlorophyll content, LWP, SDW and TDW in the SW group (Table 7). The intercellular $\mathrm{CO}_{2}$ concentration was significantly correlated with the transpiration rate, RDW, the RSR and PDR in the Ae. tauschii group, and it was significantly correlated with the three biomass-related traits and WUE in the SW group under drought conditions (Table 7). Transpiration was significantly correlated with RDW, the RSR and PDR in the Ae. tauschii group but not in the SW group.

Correlation of traits of the Ae. tauschii lines with those of the corresponding $S W$ genotypes

No morphological or physiological traits of the SW lines were significantly correlated with the corresponding traits of their parental Ae. tauschii lines under drought conditions (Table 8). Under well-watered conditions, highly significant correlations were found for the intercellular $\mathrm{CO}_{2}$ concentration and significant correlations were found for the transpiration rate, RDW, TDW and WUE. The other traits were not significantly correlated between the two groups under wellwatered conditions.

\section{SW lines from the Central Asian and Georgian groups had better performance}

The Ae. tauschii accessions were categorized into five different groups based on their geographic origin. The SW lines derived from Ae.tauschii collected from Central Asian countries and Georgia produced significantly higher TDW than in their corresponding Ae. tauschii lines and Langdon under drought condition (Fig. 1). Although not significant, these SW lines produced more TDW under well-watered conditions but less TDW under drought conditions compared to Cham 6. The Ae. tauschii accessions from West Asian countries and Iran produced higher TDW than their

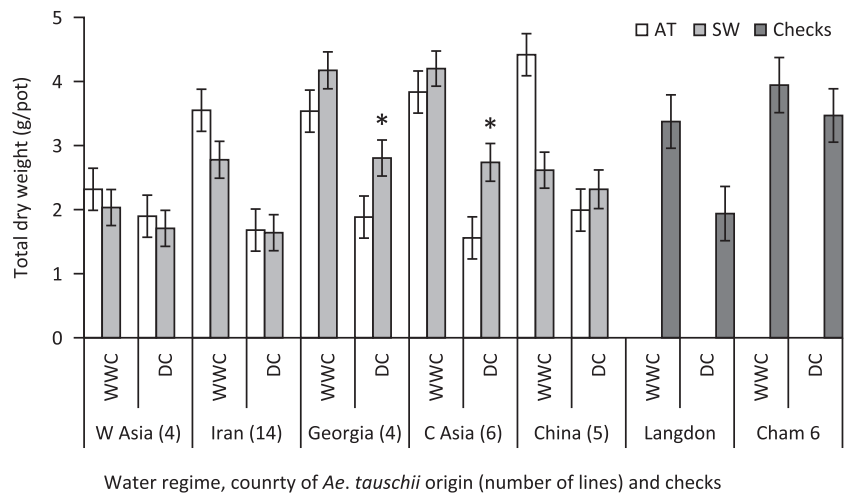

Fig. 1. Production of total dry weight of Ae. tauschii accessions and their corresponding SW based on the five geographic groups of origin of Ae. tauschii. ${ }^{*}, P<0.05$

corresponding SW lines (Fig. 1). Although the Ae.tauschii accessions from China produced the highest TDW under well-watered conditions, their corresponding SW lines produced far less TDW.

\section{Discussion}

Marked morphological and physiological variation exhibited in Ae. tauschii and SW

We observed wide and statistically significant variation in most of the morphological and physiological characteristics we measured in the Ae. tauschii accessions (Table 2). Dudnikov and Kawahara (2006) also reported wide genotypic variation among Ae. tauschii collected from regions with various agricultural and climatic conditions. These genotypic differences might result from different adaptation mechanisms to cope with unique aspects of local management techniques and environmental stresses. Therefore, the morphological and physiological variations observed in Ae. tauschii could result from wide differences in the agricultural and climatic conditions among their diverse origins. On the other hand, the SW genotypes derived from the 33 different Ae. tauschii lines exhibited wide variation in their morphological and physiological responses to drought (Table 2). These results appear to be consistent with the wide genotypic variation in their parental Ae. tauschii lines.

\section{SW showed marked drought tolerance}

The SW lines exhibited greater drought tolerance than the Ae. tauschii accessions; that is, they showed a smaller reduction in the values of most traits when exposed to drought (Table 3). In particular, the biomass-related traits (TDW, RDW and SDW) showed clearly lower reductions under drought, and the RSR and PDR values increased under drought, versus a decrease for Ae. tauschii.

Increased PDR in the SW lines under drought resulted in an increased RDW and root-shoot ratio in the SW lines, which suggests the higher capacity of these plants to exploit soil water by expanding their root distribution. These results 
are in good agreement with those of Reynolds et al. (2007), who reported that SW lines performed better under moisture stress due to their increased partitioning of root mass to deeper soil layers and their increased ability to extract moisture from deeper depths.

Higher WUE is considered crucial for resistance to drought stress. In this study, WUE provided the clear difference in drought tolerance among the genotypes. The SW lines used water more efficiently than the Ae. tauschii lines under drought conditions and showed a much smaller reduction compared with well-watered conditions (Table 3). Some SW lines such as SW5, SW2 and SW19 had a higher WUE $\left(3.3,3.0\right.$ and $3.0 \mathrm{~g} \mathrm{~kg}^{-1}$, respectively) than the standard drought-tolerant cultivar Cham $6\left(2.3 \mathrm{~g} \mathrm{~kg}^{-1}\right)$. Our results are in line with those of Huang et al. (2007), who reported increased WUE at higher ploidy levels. Higher yield is associated with high WUE (Angus and van Herwaarden 2001), therefore these SW lines may represent useful resources for breeding high-yield cultivars.

The overall photosynthetic rates of the Ae. tauschii lines were higher than those of the SW lines under both drought and well-watered conditions (Table 3), which agrees with earlier reports that Ae. tauschii was capable of maintaining a satisfactory rate of net photosynthesis even at lower relative soil water contents (Dulai et al. 2006, Wang et al. 2008).

Some SW lines were more drought-tolerant than the standard cultivar (Cham 6), and these lines may be good candidates for breeding programs to develop drought-tolerant cultivars (Reynolds et al. 2005). Other lines were less droughtresistant, but showed better physiological traits that are associated with drought tolerance; these lines may still be useful materials if those traits can be transferred into commercial wheat cultivars, as suggested by van Ginkel and Ogbonnaya (2007). Wild species are not always tolerant or resistant to certain stresses, but they may still have the potential to create tolerant hexaploids. The SW lines in our study that were not drought-tolerant can still be used for breeding programs with other goals, such as rust resistance, salinity or other abiotic stresses as reported by Colmer et al. (2006), Liu et al. (2010) and Valkoun (2001).

\section{SW showed greater variation than Ae. tauschii}

In comparison with the Ae.tauschii lines, the SW genotypes generally showed more genotypic variance under drought conditions than under well-watered conditions (Table 4), and the differences were often large (more than $300 \%$ ). Moreover, except for the photosynthetic rate, intercellular $\mathrm{CO}_{2}$ concentration, and transpiration rate, the SW group had higher variation in all morphological and physiological traits under both conditions.

The Euclidean distance revealed great genetic diversity between the two groups under both conditions (Table 5). Greater variation was observed in the SW lines than in the Ae. tauschii lines under both conditions. Moreover, the SW lines exhibited more variation under drought than under well-watered conditions. These results reflect the ability of the SW lines to cope with drought conditions, which represents variation that could be exploited in breeding programs. Despite this rich diversity, not all of the SW lines would be useful, since some showed less variation in the photosynthetic rate, intercellular $\mathrm{CO}_{2}$ concentration, and transpiration rate (Table 4$)$.

The interaction between the AABB and DD genomes in the SW lines may therefore have enhanced the response to drought in some lines. These results generally agree with those of Reynolds et al. (1999), who suggested an increase in the probability of favorable traits being expressed in hexaploid and tetraploid combinations.

Table 4. Genotypic variance in the SW lines (from the ANOVA variances) in comparison with the genotypic variance in the Ae. tauschii lines (standardized to a value of 100)

\begin{tabular}{|c|c|c|c|c|c|c|c|c|c|c|c|c|}
\hline $\begin{array}{l}\text { Water } \\
\text { regime }\end{array}$ & $\begin{array}{c}\text { Photosynthe- } \\
\text { sis }\end{array}$ & $\begin{array}{c}\text { Stomatal } \\
\text { conductance }\end{array}$ & $\begin{array}{l}\text { Intercellular } \\
\mathrm{CO}_{2}\end{array}$ & $\begin{array}{c}\text { Transpira- } \\
\text { tion rate }\end{array}$ & $\begin{array}{c}\text { Chlorophyll } \\
\text { content }\end{array}$ & $\mathrm{LWP}^{b}$ & RDW & SDW & TDW & RSR & PDR & WUE \\
\hline WWC & 72.0 & 110.7 & 58.4 & 30.9 & 237.4 & 103.0 & 128.6 & 133.8 & 141.6 & 66.1 & 100.6 & 168.5 \\
\hline $\mathrm{DC}$ & 82.7 & 108.1 & 76.7 & 40.7 & 131.4 & 113.4 & 387.2 & 308.8 & 430.1 & 187.6 & 124.1 & 376.4 \\
\hline
\end{tabular}

$a$ WWC, well-watered conditions; DC, drought conditions.

${ }^{b}$ LWP, leaf water potential; RDW, root dry weight; SDW, shoot dry weight; TDW, total dry weight; RSR, root-shoot ratio; PDR, partitioning of dry matter to roots (\%); WUE, water-use efficiency.

Table 5. Euclidean distance standardized to $[0,1]$ to show the morphological and physiological diversity for Ae. tauschii and SW under drought and well-watered conditions based on the averages of the 12 morphological and physiological traits shown in Tables 2 and 3

\begin{tabular}{lccccccccc}
\hline \hline Water regime $^{a}$ & Group & Mean & Minimum & Maximum & Range & Variance & SD & Median & CV \\
\hline WWC & AT $^{b}$ & 0.486 & 0.173 & 1.000 & 0.827 & 0.022 & 0.147 & 0.491 & 30.243 \\
& SW & 0.539 & 0.139 & 1.000 & 0.861 & 0.025 & 0.158 & 0.543 & 29.391 \\
\hline DC & AT & 0.475 & 0.146 & 1.000 & 0.854 & 0.020 & 0.143 & 0.464 & 30.073 \\
& SW & 0.593 & 0.175 & 1.000 & 0.825 & 0.033 & 0.180 & 0.588 \\
\hline
\end{tabular}

${ }^{a}$ WWC, well-watered conditions; DC, drought conditions; SD, standard deviation; CV, coefficient of variation.

${ }^{b}$ AT, Ae. tauschii; SW, synthetic hexaploid wheat. 


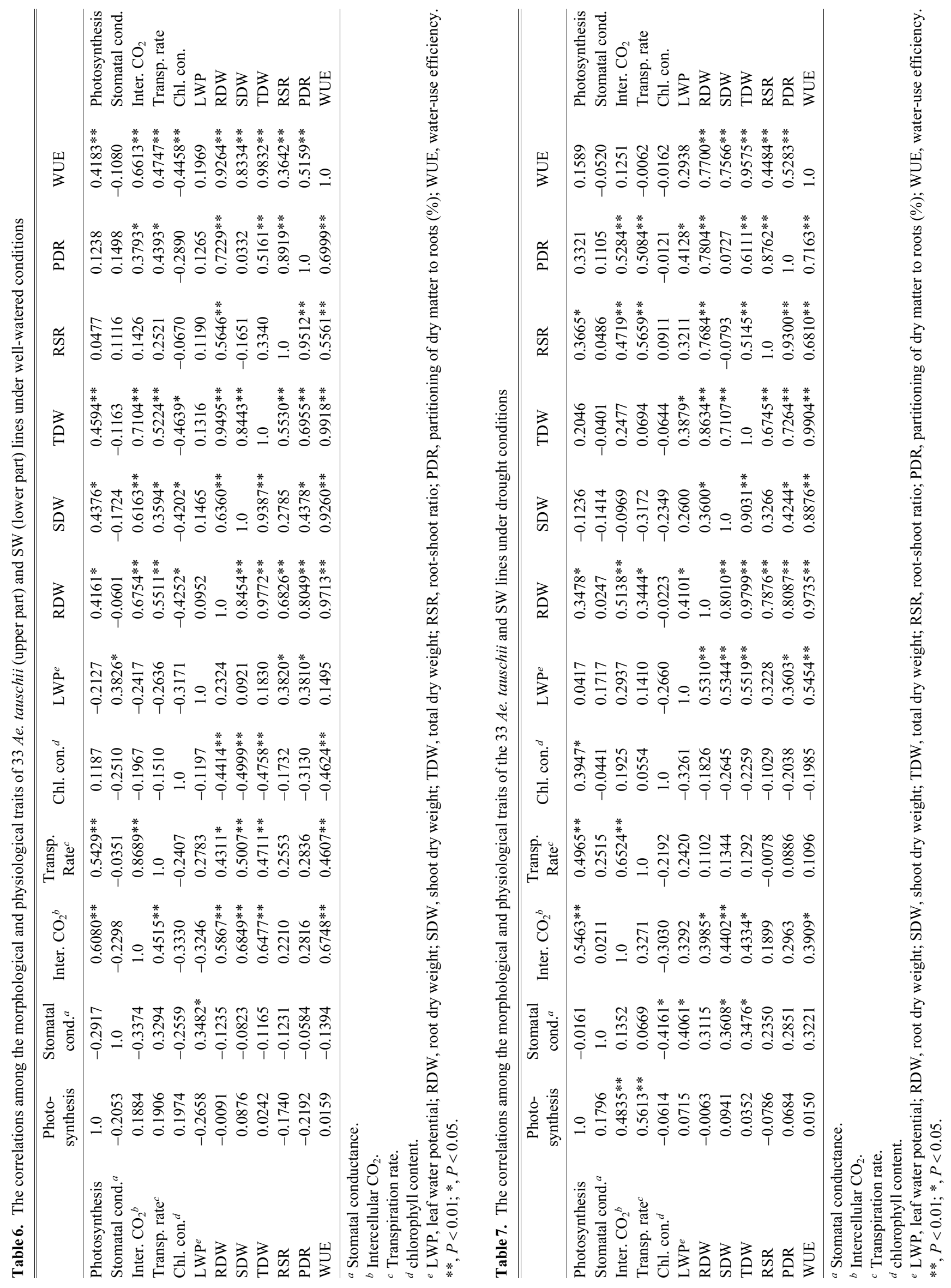


Correlations among the morphological and physiological traits

The correlations of morphological and physiological traits within the Ae. tauschii and within SW lines had some similarities under well-watered and drought conditions, but we noted many differences between the morphological and physiological traits that could indicate different interactions between the parent, genotypes and various environmental conditions. These differences need to be further explored in order to understand their potential usefulness in breeding programs.

The photosynthetic rate was significantly correlated with the intercellular $\mathrm{CO}_{2}$ concentration and transpiration rate. However, for the SW lines under well-watered conditions, this correlation was not significant (Tables 6, 7). The photosynthetic rate was not significantly correlated with stomatal conductance in both groups and under both conditions, which indicate that stomatal conductance might not be main determinant factor for photosynthetic rate under drought condition. Other possible factors such as photophosphorylation and ATP synthesis have been suggested to affect and limit photosynthetic under drought (Medrano et al. 2002).

Stomatal conductance was significantly correlated with LWP in both groups under well-watered conditions (Table 6), but under drought condition a significant correlation was found only in SW group. Under water stress, a good correlation is often observed between leaf water potential and stomatal conductance (Medrano et al. 2002). These results indicate the importance of stomatal conductance interaction with LWP for drought tolerance in SW.

WUE was significantly correlated with RDW, SDW, TDW, the root-shoot ratio, and PDR in both groups and under both conditions (Tables 6,7 ), which indicates the importance of the WUE in maximizing total biomass in these species regardless of the water availability.

Correlation of the Ae. tauschii lines with their corresponding $S W$ genotypes

We found no correlations between the Ae.tauschii lines and their corresponding SW lines for any of the studied traits under drought conditions while under well watered condition intercellular $\mathrm{CO}_{2}$ concentration, transpiration rate, RDW, TDW and WUE were correlated. Kajimura et al. (2011) reported that heading, flowering and maturation time of Ae. tauschii lines correlated with their corresponding SW lines (Table 8). This indicates that the performance of the SW lines appeared to result from complex interactions between the potential of Ae.tauschii itself and the genes in durum wheat, as was reported by Trethowan et al. (2005). These results suggest that the Ae. tauschii lines and their corresponding SW lines exhibit nearly independent behavior under different conditions. Our results provide supporting evidence for the findings of Fujiwara et al. (2010) and Kurahashi et al. (2009), who found that the genome variations observed at the diploid genome level (DD) were not necessarily reflected at the hexaploid level in the SW lines.
Table 8. Correlations between the morphological and physiological traits of the Ae. tauschii lines and their corresponding SW lines under well-watered and drought conditions

\begin{tabular}{lrc}
\hline \hline Trait $^{b}$ & Under DC $^{a}$ & Under WWC \\
\hline Photosynthesis & $0.0247 \mathrm{~ns}$ & $0.0920 \mathrm{~ns}$ \\
Stomatal conductance & $-0.1072 \mathrm{~ns}$ & $0.1474 \mathrm{~ns}$ \\
Intercellular $\mathrm{CO}_{2}$ & $0.3198 \mathrm{~ns}$ & $0.4877 * *$ \\
Transpiration rate & $0.0536 \mathrm{~ns}$ & $0.3686 *$ \\
Chlorophyll content & $0.3371 \mathrm{~ns}$ & $0.0655 \mathrm{~ns}$ \\
$\mathrm{LWP}^{b}$ & $0.1996 \mathrm{~ns}$ & $-0.2439 \mathrm{~ns}$ \\
RDW & $0.3049 \mathrm{~ns}$ & $0.3576 *$ \\
SDW & $0.1473 \mathrm{~ns}$ & $0.2482 \mathrm{~ns}$ \\
TDW & $0.2881 \mathrm{~ns}$ & $0.3629 *$ \\
RSR & $0.1149 \mathrm{~ns}$ & $-0.0251 \mathrm{~ns}$ \\
PDR & $0.1498 \mathrm{~ns}$ & $0.1721 \mathrm{~ns}$ \\
WUE & $0.2430 \mathrm{~ns}$ & $0.3634 *$ \\
\hline
\end{tabular}

${ }^{a}$ WWC, well-watered conditions; DC, drought conditions.

${ }^{b}$ LWP, leaf water potential; RDW, root dry weight; SDW, shoot dry weight; TDW, total dry weight; RSR, root-shoot ratio; PDR, partitioning of dry matter to roots (\%); WUE, water-use efficiency; LSD, least significant difference.

**, $P<0.01 ; *, P<0.05$; ns, not significant.

\section{SW lines from the Central Asian and Georgian groups had enhanced performance}

We divided the Ae. tauschii and SW genotypes into five groups based on their origin. The SW lines developed from Ae. tauschii accessions from Georgia and Central Asia performed better than their corresponding Ae. tauschii genotypes under both water regimes (Fig. 1). In contrast, the Ae. tauschii accessions from West Asia performed better than their corresponding SW lines under both conditions. This emphasizes the importance of utilizing the genetic resources from each region judiciously when breeding wheat for drought resistance.

We suggest that, regardless of the adaptability and performance of the Ae. tauschii lines under drought stress, the production of many SW lines would probably result in genotypes with enhanced trait expression due to gene interactions. One way to take advantage of this useful variation would be to use the synthetic wheat derivatives system proposed by Tsujimoto et al. (2011). Based on the results of the present study, we recommend that the useful traits of the SW lines observed at the hexaploid level be used not only for improving drought tolerance but also for breeding wheat cultivars capable of resisting other biotic and abiotic stresses.

\section{Acknowledgments}

We thank J. Valkoun (ICARDA), J. Konopka (ICARDA), H. Bockelman (USDA), A. Graner (IPK) and K. Kato (Okayama University) for Ae. tauschii accession seeds.

\section{Literature Cited}

Allen,C.D., A.K.Macalady, H.Chenchouni, D.Bachelet, N.McDowell, 
M.Vennetier, T.Kitzberger, A.Rigling, D.D.Breshears, E.H.Hogg et al. (2010) A global overview of drought and heat-induced tree mortality reveals emerging climate change risks for forests. For. Ecol. Manage. 259: 660-684.

Angus,J.F. and A.F.van Herwaarden (2001) Increasing water use and water use efficiency in dryland. Wheat Agron. J. 93: 290-298.

Aprile, A., A.M. Mastrangelo, A.M. De Leonardis, G. Galiba, E. Roncaglia, F.Ferrari, L.De Bellis, L.Turchi, G.Giuliano and L. Cattivelli (2009) Transcriptional profiling in response to terminal drought stress reveals differential responses along the wheat genome. BMC Genomics 10: 279. doi: 10.1186/1471-2164-10-279.

Ashraf,M. (2010) Inducing drought tolerance in plants: Recent advances. Biotechnol. Adv. 28: 169-183.

Assefa, S. and H.Fehrmann (2000) Resistance to wheat leaf rust in Aegilops tauschii Coss. and inheritance of resistance in hexaploid wheat. Genet. Resour. Crop. Evol. 47: 135-140.

Battisti,S. and R.L.Naylor (2009) Historical warnings of future food insecurity with unprecedented seasonal heat. Science 323: 240-244.

Bloem,M.W., R.D.Semba and K.Kraemer (2010) Castel Gandolfo workshop: an introduction to the impact of climate change, the economic crisis and the increase in the food prices on malnutrition. J. Nutr. 140: 132S-135S.

Carnicer,J., M.Coll, M.Ninyerola, X.Pons, G.Sánchez and J.Peñuelas (2010) Wide spread crown condition decline, food web disruption, and amplified tree mortality with increased climate change-type drought. Proc. Natl. Acad. Sci. USA 108: 1474-1478.

Colmer,T.D., J.Flowers and R.Munns (2006) Use of wild relatives to improve salt tolerance in wheat. J. Exp. Bot. 57: 1059-1078.

Cox,T.S., W.J.Raupp and B.S.Gill (1994) Stem rust resistance $L R 41$, $L R 42$, and LR43 transferred from Triticum tauschii to common wheat. Crop Sci. 34: 339-343.

Dudnikov, A.J. and T.Kawahara (2006) Aegilops tauschii: genetic variation in Iran. Genet. Resour. Crop. Ev. 53: 579-586.

Dulai,S., I.Molnar, J.Pronay, A.Csernak, R.Tarnai and M.MolnarLang (2006) Effects of drought on photosynthetic parameters and heat stability of PSII in wheat and in Aegilops species originating from dry habitats. Acta Biol. Szeged. 50: 11-17.

Entz,M.H. and D.B.Fowler (1990) Differential agronomic response of winter wheat cultivars to pre-anthesis environmental stress. Crop Sci. 30: 1119-1123.

Feldman,M. (2001) Origin of cultivated wheat. In: Bonjean,A.P. and W.J.Angus (eds.) The World Wheat Book: A History of Wheat Breeding, Lavoisier Publishing, Paris, pp. 3-53.

Fujwara,Y., S.Shimada, S.Takumi and K.Mura (2010) Differential effects of Aegilops tauschii genotypes on maturing-time in synthetic hexaploid wheats. Breed. Sci. 60: 286-292.

Helbaek,H. (1959) Domestication of food plants in the old world. Science 130: 365-372.

Huang, M., X.Deng, Y.Zhao, S.Zhou, S.Inanaga, S. Yamada and K. Tanaka (2007) Water and nutrient use efficiency in diploid, tetraploid and hexaploid wheats. J. Integr. Plant. Biol. 49: 706-715.

Kajimura,T., K.Murai and S.Takumi (2011) Distinct genetic regulation of flowering time and grain-filling period based on empirical study of D-genome diversity in synthetic hexaploid wheat lines. Breed. Sci. 61: 130-141.

Kihara,H. (1944) Discovery of the DD-analyser, one of the ancestors of Triticum vulgare. Agric. Hort. 19: 13-14.

Kurahashi,Y., A.Terashima and S.Takumi (2009) Variation in dehydration tolerance, ABA sensitivity and related gene expression patterns in D-genome progenitor and synthetic hexaploid wheat lines. Int. J. Mol. Sci. 10: 2733-2751.
Liu,D., L.Zhang and Z.Yan (2010) Stripe rust resistance in Aegilops tauschii and its genetic analysis. Genet. Resour. Crop. Evol. 57: 325-328.

Matsuoka,Y. and S.Nasuda (2004) Durum wheat as a candidate for the unknown female progenitor of bread wheat: an empirical study with a highly fertile $\mathrm{F}_{1}$ hybrid with Aegilops tauschii Coss. Theor. Appl. Genet. 109: 1710-1717.

Matsuoka,Y., S.Takumi and T.Kawahara (2007) Natural variation for fertile triploid $\mathrm{F}_{1}$ hybrid formation in allohexaploid wheat speciation. Theor. Appl. Genet. 115: 509-518.

Medrano,H., J.M.Escalona, J.Bota, J.Gulias and J.Flexas (2002) Regulation of photosynthesis of $\mathrm{C}_{3}$ plants in response to progressive drought: Stomatal conductance as a reference parameter. Ann. Bot. 89: 895-905.

Mujeeb-Kazi,A., V.Rosas and S.Roldan (1996) Conservation of the genetic variation of Triticum tauschii in synthetic hexaploid wheats and its potential utilization for wheat improvement. Genet. Resour. Crop Evol. 43: 129-134.

Naghavi,M.R. and M.Mardi (2010) Characterization of genetic variation among accessions of Aegilops tauschii. AsPac J. Mol. Biol. Biotechnol. 18: 93-96.

Rajaram, S. (2001) Prospects and promise of wheat breeding in the 21st century. Euphytica 119: 3-15.

Reif,J.C., P.Zhang, S.Dreisigacker, M.L.Warburton, M.van Ginkel, D.Hoisington, M.Bohn and A.E.Melchinger (2005) Wheat genetic diversity trends during domestication and breeding. Theor. Appl. Genet. 110: 859-864.

Reynolds, M., F.Dreccer and R.Trethowan (2007) Drought-adaptive traits derived from wheat wild relatives and landraces. J. Exp. Bot. 58: $177-186$

Reynolds, M.P., B.Skovmand, R.Trethowan and W.Pfeiffer (1999) Evaluating a conceptual model for drought tolerance. In: Ribaut, J.M. (ed.) Using Molecular Markers to Improve Drought Tolerance, International Maize and Wheat Improvement Center (CIMMYT), Mexico City, Mexico, pp. 49-53.

Reynolds,M.P., A.Mujeeb-Kazi and M.Sawkins (2005) Prospects for utilising plant-adaptive mechanisms to improve wheat and other crops in drought and salinity prone environments. Ann. Appl. Biol. 146: 239-259.

Shirazi,M.U., J.A.Gyamfi, T.Ram, H.Bachiri, B.Rasyid, A.Rehman, M.A.Khan, S.M.Mujtaba, M.Ali, A.Shreen et al. (2010) Selection of some suitable drought tolerant wheat genotypes using carbon isotopes discrimination (CID) technique. Pak. J. Bot. 42: 36393644.

Soussana,J.F., A.I.Graux and F.N.Tubiello (2010) Improving the use of modeling for projections of climate change impacts on crops and pastures. J. Exp. Bot. 61: 2217-2228.

Takumi,S., Y.Naka, H.Morihiro and Y.Matsuoka (2009) Expression of morphological and flowering time variation through allopolyploidization: an empirical study with 27 wheat synthetics and their parental Aegilops tauschii accessions. Plant Breed. 128: 585-590.

Teknomo,K. (2011) Similarity Measurement. http://people.revoledu. com/kardi/tutorial/Similarity/ accessed on April 7, 2011.

Trethowan, R.M., M. Reynolds, K. Sayre and I. Ortiz-Monasterio (2005) Adapting wheat cultivars to resource conserving farming practices and human nutritional needs. Ann. Appl. Biol. 146: 405413.

Tsujimoto,H., S.Wang, M.Garg and H.Tanaka (2011) Alien genetic resources for dryland wheat breeding. In: El-Beltagy, A.S. and M.Saxina (eds.). Meeting the Challenge of Sustainable Development in Drylands under Changing Climate. Proceedings of the $10^{\text {th }}$ 
International Conference on Development of Drylands, International Dryland Development Commission (IDDC), Cairo (in press).

Valkoun,J.J. (2001) Wheat pre-breeding using wild progenitors. Euphytica 119: 17-23.

van Ginkel,M., D.S.Calhoun, G.Gebeyeho, A.Mirenda, C.Tain-you, R.Pargas Lara, R.M.Trethowan, K.Saure, J.Crossa and S.Rajaram (1998) Plant traits related to yield of wheat in early, late or continuous drought conditions. Euphytica 100: 109-121.

van Ginkel,M. and F.Ogbonnaya (2007) Novel genetic diversity from synthetic wheats in breeding cultivars for changing production conditions. Field Crops Res. 104: 86-94. van Slageren,M.W. (1994) Wild wheats: a monograph of Aegilops L. and Amblyopyrum (Jaub. et Spach) Eig (Poaceae). ICARDA and Wageningen Agric. Univ. 94, p. 512.

Wang,C., M.Li, J.Song, Y.Chi, X.Wang and Y.Wu (2008) Differences in stomatal and photosynthetic characteristics of five diploidy wheat species. Acta Ecol. Sin. 28: 3277-3283.

Webb,P. (2010) Medium to long run implications of high food prices for global nutrition. J. Nutr. 140: 143S-147S.

Zaharieva, M., P. Monneveux, M.Henry, R. Rivoal and J.Valkoun (2001) Evaluation of a collection of wild wheat relative Aegilops geniculata Roth and identification of potential sources for useful traits. Euphytica 119: 33-38. 